"Theory of Auger neutralization of ions at the surface of a diamond-type semiconductor," Phys. Rev., vol. 122, pp. 83-113, $1961 ;$ - "Excited-atom deexcitation spectroscopy using incident ions," Phys. Rev. Lett., vol. 43, pp. 1050-1053, 1979.

[10] W. C. Murphy, A. C. Beri, T. F. George, and J. Lin, "Analysis of laser-enhanced adsorption/desorption processes on semiconductor surfaces via electronic surface state excitation," in Proc. Mat. Res. Soc. Symp., vol. 17, pp. 273-282, 1983.

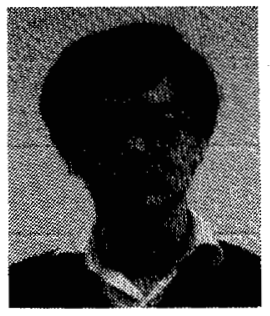

Hai-Woong Lee was born in Seoul, Korea, in 1947. He received the B.S. degree from Seoul National University in 1970 and the Ph.D. degree from the University of Pittsburgh, Pittsburgh, PA, in 1977.

After doing postdoctoral work at the University of Rochester, Rochester, NY, the University of Arizona, Tucson, and the University of New Mexico, Albuquerque, he joined the faculty of Oakland University, Rochester, MI, in 1981 where he is an Assistant Professor of Physics. His research activities include theoretical quantum optics and collision theory.
Dr. Lee is a member of the American Physical Society, the Optical Society of America, and Sigma Xi.

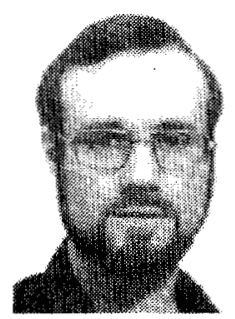

Thomas F. George was born in Philadelphia, PA, on March 18, 1947. He received the B.A. degree in chemistry and mathematics from Gettysburg College, Gettysburg, PA, in 1967, and the M.S. and Ph.D. degrees in chemistry from Yale University, New Haven, CT, in 1968 and 1970 , respectively.

During 1970-1971 he was a Research Associate at the Massachusetts Institute of Technology, Cambridge, and during 1971-1972 he was a Postdoctoral Appointee at the University of California, Berkeley. In September 1972 he joined the staff of the University of Rochester, Rochester, NY, as Assistant Professor of Chemistry. He became an Associate Professor in 1974 and a Professor in 1977. His research interests include molecular energy transfer, reaction dynamics, and the interaction of laser radiation with molecular rate processes in the gas phase and at a solid surface.

Dr. George is a member of the American Chemical Society, the American Physical Society, the Royal Society of Chemistry, the Society of Photo-Optical Instrumentation, and is a Fellow of the New York Academy of Sciences. He is on the Advisory Board of the Journal of Physical Chemistry:

\title{
Table-Top EUV Continuum Light Source
}

\author{
P. K. CARROLL, EUGENE T. KENNEDY, AND GERARD O'SULLIVAN
}

\begin{abstract}
Work in recent years on the development of a convenient "table-top" source of continuum radiation in the XUV and VUV from 4 to $200 \mathrm{~nm}$ is summarized. It was found that laser-produced plasmas on targets of high atomic number $(62 \leqslant Z \leqslant 74)$ emitted apparently line-free continua over very substantial spectral regions in the XUV and VUV. The continua are very intense, reproducible, and relatively insensitive to ambient pressure. The effective absence of line emission can be explained on the basis of the electron configurations of the ionized species responsible for the emission. A high repetition rate modular version of the source is described. Applications and advantages of the light source are presented.
\end{abstract}

\section{INTRODUCTION}

$\mathrm{T}$ HE development of the synchrotron as a source of continuum radiation has led, in recent years, to a considerable growth of activity in such areas as absorption spectroscopy and photoionization in the VUV and the XUV regions. Although very effective as a light source, the synchrotron has the extreme disadvantages of being very costly and nonportable, and the need for a much simpler and cheaper light source is very apparent. We believe that the simple compact light

Manuscript received February 7, 1983; revised September 2, 1983. This work was supported in part by the National Board for Science and Technology (Ireland) under Research Grant URG/143/82 and by the NSF under Grant PHT 80-16657.

P. K. Carroll is with the Department of Physics, University College, Dublin, Ireland.

E. T. Kennedy and G. O'Sullivan are with the School of Physical Sciences, National Institute for Higher Education, Dublin, Ireland. source described in the present paper and which was developed initially in the Department of Physics, University College, Dublin, Ireland, has much to offer and indeed has been used successfully on a routine basis as a table-top source in our laboratory $[1],[2]$.

\section{EARLY EXPERIMENTS WITH Laser-Produced Continua}

Shortly after the development of the giant pulse $Q$-switch laser it was found that when its output was focused on a solid target material in vacuum, a dense high-temperature plasma was generated. The spectra of such plasmas were found typically to originate in species in high stages of ionization. Many new spectra were generated and studied in this way; as typical examples, we quote the work of Fawcett [3] and Doschek et al. [4]. In all these cases the spectra were dominated by strong line emission and the thrust of the work was on conventional term analysis. To a greater or lesser extent continuum originating in recombination and bremsstrahlung was always present. Because of the dominance of strong lines, however, the continuum was quite unsuitable as a background for absorption studies.

A systematic study of target materials was undertaken in this laboratory with the hope of finding a spectrum in which the lines would be, to a greater or lesser degree, suppressed with respect to the continuum. In the course of this work almost all the heavier elements from strontium $(Z=38)$ to uranium 
$(Z=92)$ were investigated. The exciting laser was a $Q$-switched ruby system which gave an output of $1 \mathbf{J}$ in a pulse of about 25 ns. In the early experiments several target materials were mounted in a small chuck which could be rotated in front of the spectrograph slit, so that a number of spectra could be obtained in a single experiment. Most of the observations were made in the region of $4-50 \mathrm{~nm}$, as it was in the range below $50 \mathrm{~nm}$ that the greatest need for a good continuum source existed. The instrument used was a $2 \mathrm{~m}$ grazing incidence vacuum spectrograph which had a grating with 1200 grooves $/ \mathrm{mm}$ blazed at $6.5 \mathrm{~nm}$. A number of the more promising spectra were also studied in the region of $50-280 \mathrm{~nm}$ with a $3 \mathrm{~m}$ normal incidence instrument blazed at $150 \mathrm{~nm}$.

Most of the spectra studied showed strong line emission accompanied by the usual continuum background. As one moved through the rare earths, however, the importance of the line contribution decreased and the spectra became more continuum-like. In particular, the spectra from samarium to ytterbium were almost completely free from lines throughout the vacuum region from $4 \mathrm{~nm}$ to longer wavelengths [1], [2] . Such lines as did occur were relatively weak and lay for the most part at the longer wavelength end of the spectrum. Lutetium and hafnium also gave good continua but in these instances the tendencies for lines to appear at longer wavelengths became more pronounced. In terms of freedom from lines in the longer wavelength region, i.e., up to about $200 \mathrm{~nm}$, the best target material in our experiments was samarium. In the shorter wavelength region (below $50 \mathrm{~nm}$ ) the elements from terbium to ytterbium were particularly good. (It should perhaps be mentioned here that below $12 \mathrm{~nm}$ tungsten gave a good continuum but had many strong lines at longer wavelengths.) The spectra were almost completely line free over the regions mentioned and also exhibited a smooth variation of spectral intensity with some broad regions of enhancement, particularly in the range below $20 \mathrm{~nm}$.

\section{Improved Automatic Light Source}

To render the operation of the source consistent, it is desirable that a new element of target surface be presented to the laser beam after a limited number of shots. This is necessary because of pitting of the target surface; the number of shots which can be sustained, typically 1-5, depends on the laser power and the target material. An automatic target chamber was developed to meet these needs. In the case of ytterbium, a foil of the metal was wrapped on a cylindrical mandrel about $1.6 \mathrm{~cm}$ in diameter and $5 \mathrm{~cm}$ long. By using a tapped flange on top of the vacuum chamber and a rotary vacuum seal on the bottom, the cylinder was rotated and translated by a $0.9^{\circ}$ stepper motor so that the surface of the target was scanned in a spiral of pitch $0.64 \mathrm{~mm}$. The motor was triggered by a photoelectric signal picked up from the laser-produced plasma and processed by logic control and driver circuitry. The system could be run at repetition rates up to $50 \mathrm{pps}$ and provision was made in the logic circuitry to skip the seam in each revolution of the target. Some details of the target chamber design are shown in Fig. 1. Preliminary experiments with this light source are described in [5].
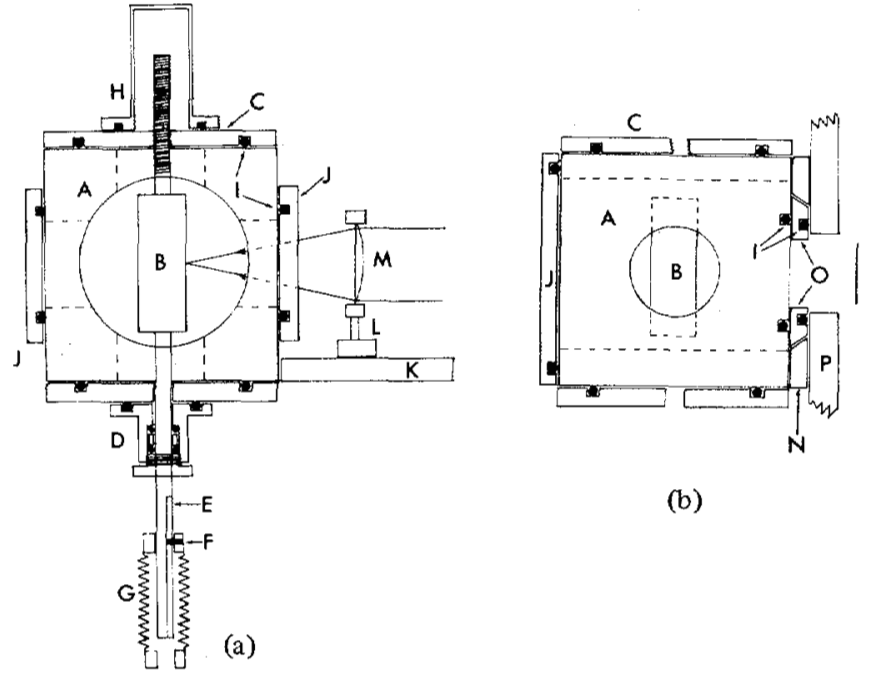

(b)

Fig. 1. Automatic pulsed light source. (a) Back view (b) Side view. Some detail shown in (a) is not repeated in (b). $A$ : Aluminum cube $(8.25 \mathrm{~cm}$ side) machined and drilled to house target. $B$ : Cylindrical target mounted on stainless steel drive shaft. $C$ : Threaded flange for vertical translation. $D$ : Rotary vacuum seal. $E$ : Slot in shaft. $F$ : Driving pin. $G$ : Flexible lingage to stepper motor. $H$ : Vacuum cap. I: $O$-ring seals. $J$ : Windows. $K$ : Lens mount attached rigidly to target chamber. $L$ : Moveable lens holder. $M$ : Lens to focus laser beam. $N$ : Bevelled ways attached to target chamber. $O$ : Bevelled plate attached to spectrograph. $P$ : Spectrograph wall. $Q:$ Spectrograph slit. The plate $O$ can be rotated through $90^{\circ}$ so that the plasma plume may be viewed in a direction parallel, as well as perpendicular, to the slit.

\section{Optimization of Light Output}

It has been demonstrated that the VUV output from the plasma depends on the angle of incidence $\phi$ and the angle of observation $\psi$ measured with respect to the target normal (Fig. 2). For a gadolinium target irradiated by a $2.2 \mathrm{~J}, 30 \mathrm{~ns}$ ruby laser such that $\phi+\psi=90^{\circ}$, the output at $138 \mathrm{~nm}$ was optimum for $\phi=\psi=45^{\circ}[6]$. These results are in general agreement with those of Breton and Papoular [7] who observed little difference in the emitted energy for values of $\phi$ of up to $45^{\circ}$ for $\psi=30^{\circ}$ with a $0.8 \mathrm{~J}$ ruby pulse, and found that the plasma should be viewed within $50^{\circ}$ of the target normal for optimum light output. Thus, for maximum output, it is recommended that the incident laser light and viewing directions are each at $\sim 45^{\circ}$ to the target normal as shown in Fig. 2.

\section{INTENSITY}

Laser-produced plasmas have, when averaged over their short pulse lengths, the highest spectral radiance of any UV/soft Xray source. The spectral irradiances were measured with gadolinium and ytterbium targets in the $110-220 \mathrm{~nm}$ spectral region [6] for plasmas generated by a $2.2 \mathrm{~J}, 30$ ns ruby laser pulse at a distance of $50 \mathrm{~cm}$ from the source. For ytterbium the measured irradiance was $2 \times 10^{4} \mu \mathrm{W} \cdot \mathrm{cm}^{-2} \cdot \mathrm{nm}^{-1}$ at 120 $\mathrm{nm}$ and decreased monotonically to $4 \times 10^{3} \mu \mathrm{W} \cdot \mathrm{cm}^{-2} \cdot \mathrm{nm}^{-1}$ at $200 \mathrm{~nm}$. For gadolinium it had a similar distribution but was on average greater by a factor of 2 . As the gadolinium target was plane while the ytterbium was cylindrical, the difference in measured irradiance was attributed to the different geometries. The plasma diameter at $120 \mathrm{~nm}$ was estimated from spectra taken with a $3 \mathrm{~m}$ normal incidence spectrograph under astigmatic focal conditions and was found to be $\sim 125$ 


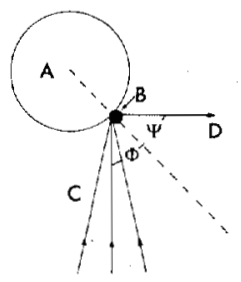

Fig. 2. Geometry of target illumination and observation. A: Target. $B$ : Plasma. $C$ : Light from laser. $D$ : Direction of light entering spectrograph.

$\mu \mathrm{m}$. Combining these data we find the spectral radiance for ytterbium at $120 \mu \mathrm{m}$ to be $2 \times 10^{5} \mathrm{~W} \cdot \mathrm{cm}^{-2} \cdot \mathrm{nm}^{-1} \cdot \mathrm{sr}^{-1}$ or $1 \times 10^{22}$ photons $\cdot \mathrm{cm}^{-2} \cdot \mathrm{sr}^{-1} \cdot \mathrm{s}^{-1}$. The peak emission from these plasmas, as determined photographically, occurs in the 10-12 $\mathrm{nm}$ region, to which our absolute irradiance measurements do not extend. At shorter wavelengths still, however, Nicolosi et al. [8] measured the spectral radiance of plasmas generated on a variety of target materials including tungsten using a $10 \mathrm{~J}, 15 \mathrm{~ns}$ ruby laser. At this increased laser power the values decrease fairly smoothly from a peak of $6 \times 10^{8}$ $\mathrm{W} \cdot \mathrm{cm}^{-2} \cdot \mathrm{sr}^{-1} \cdot \mathrm{nm}^{-1}$ at $3 \mathrm{~nm}$ to $6 \times 10^{7} \mathrm{~W} \cdot \mathrm{cm}^{-2} \cdot \mathrm{sr}^{-1} \cdot$ $\mathrm{nm}^{-1}$ at $8 \mathrm{~nm}$ which corresponds to $\sim 10^{25}$ photons $\cdot \mathrm{cm}^{-2}$. $\mathrm{sr}^{-1} \cdot \mathrm{nm}^{-1} \cdot \mathrm{s}^{-1}$ throughout this range. In our experiments the laser energy was typically $1 \mathrm{~J}$, and since above this energy the output is relatively insensitive to incident laser flux (under constant focus) [6], a radiance of approximately $10^{7} \mathrm{~W} \cdot$ $\mathrm{cm}^{-2} \cdot \mathrm{sr}^{-1} \cdot \mathrm{nm}^{-1}$ would be expected at $8 \mathrm{~nm}$. In this connection it should be noted that the output does not depend strongly on focusing conditions (for constant laser flux) [9] . We therefore expect the conditions for increased radiance to be a simultaneous increase in laser energy and focal spot size.

\section{Physics of the SOURCE}

Applications of the Colombant and Tonon model [10] to the plasmas under discussion have shown them to have an electron density close to the cutoff density of ruby light $(2 X$ $10^{21} \mathrm{~cm}^{3}$ ) [11]. Under these conditions, ion stages up to and including the sixteenth are present in the plasmas. Because of the opacity of the plasma to line radiation, recombination would be expected to be the dominant radiative process, especially at shorter wavelengths. (For $\mathrm{CO}_{2}$ plasmas, however, because of the lower ion density, line radiation predominates). Bremsstrahlung contributes $10-20$ percent of the overall continuum intensity and is most important at longer wavelengths. The absence of lines from a ruby plasma generated in rare earth materials, however, cannot be explained completely by opacity arguments since strong line radiation is always observed from targets of both lower and higher $Z$ materials. To understand the apparent absence of line radiation in the cases mentioned requires a detailed consideration of the electron configurations of the ions involved.

In low ion stages of the elements $62 \leqslant Z \leqslant 74$ the $5 s$ and $5 p$ subshells are complete while the $4 f$ contain a variable number of electrons, depending on $Z$ and ion stage. With increasing ionization the binding energy of the $4 f$ level increases much more rapidly than that of the $5 p$ or $5 s$ so that the $4 f$ level crosses the $5 p$ near to the sixth ion stage and the $5 s$ past the thirteenth [12]. As a result, each of the elements of interest contain $4 f$ electrons in their ground states for all ion stages generated. Furthermore, because of the proximity of $4 f, 5 p$, and $5 s$ binding energies, a large number of complex configurations containing variable numbers of $5 p, 5 s$, and $4 f$ electrons lie within a narrow energy bandwidth. Thus, a simple xenonlike configuration $5 s^{2} 5 p^{6}$ can, further along the isoelectronic sequence, give rise to a range of configurations of the type $5 s^{m} 5 p^{k} 4 f^{n}, m+k+n=8, m=0,1,2 ; 0 \leqslant k \leqslant 6 ; 0 \leqslant n \leqslant 8$, i.e., 21 closely spaced configurations. For example, in Sm IX the ground state is calculated to be $5 s^{2} 5 p^{2} 4 f^{4}$ and the total energy spread of the configurations is $63 \mathrm{eV} \mathrm{[13].} \mathrm{Transitions}$ based on these states are extremely complex and when the full range of available low-lying states are included can give rise to hundreds of thousands of lines. As a result, the oscillator strength is so weakened that these lines are for the most part submerged in the background continuum. The strongest transitions are of the type $4 d-4 f$ and these overlap in ion stages VII-XVI to yield an unresolved transition array (UTA) about $30 \mathrm{eV}$ wide superimposed on the continuum which causes some intensity modulation in the $70 \mathrm{~nm}$ region. Again, because of the complexity of the configurations, no discrete lines are observed. To illustrate this point, the total number of allowed lines predicted for a $4 d^{10} 4 f^{6}-4 d^{9} 4 f^{7}$ transition is 83024 and for $4 d^{10} 4 f^{3}-4 d^{9} 4 f^{4}$ is 4574 .

\section{Advantages of Laser-Produced Continua}

Before proceeding to describe some applications of the source we will briefly summarize some of its advantages.

Ease of Production: The continuum-emitting plasma is produced simply by focusing a $Q$-switched laser pulse into a suitable target. (See Sections II and III.)

Ease of Location: The beam-guiding optics and focusing lens may be easily adjusted to locate the plasma exactly where desired.

Purity: The continua in particular those emitted by the higher $Z$ rare earth plasmas are remarkably free from undesirable line emission.

Wide Spectral Coverage: Rare earth plasmas emit continua covering the range 3-200 $\mathrm{nm}$. (The short wavelength limit in the present work was, in fact, set by the performance of the spectrograph.)

Noiseless: Since the energy is delivered to the plasma in the form of light electrical pickup, problems can be eliminated.

Short Pulse Duration: The continuum pulse profile closely follows the exciting laser pulse profile having a FWHM of 30 ns for a conventional $Q$-switched laser.

Easy Synchronization: By directing a small fraction of the laser pulse onto a fast photodiode, synchronization of the continuum-emitting plasma with other events can be achieved.

Small Spatial Extent: The continuum is emitted from a very small volume close to the target surface. The point-like character of the source is of value in experiments requiring spatial resolution.

Insensitivity to Ambient Pressure: The insensitivity of the laser-produced plasma source to ambient pressures up to several torr makes it unique among far UV/soft X-ray sources and eliminates the need for differential pumping in many experiments. 
Reproducibility: The source is very stable. A shot-to-shot reproducibility to better than 10 percent was measured in early work [2] and more recently a reproducibility to 5 percent was reported [9].

High Radiance: The laser-produced plasma source has the highest instantaneous radiance of any far UV/soft X-ray source.

\section{ViII. Applications of Laser-Produced Continua}

Because of their versatility, laser-produced continua have potential applications in a number of areas.

\section{A. Absorption Spectroscopy}

The continua proved very satisfactory for absorption studies and spectra illustrating their use were presented in the original publications [1], [2]. They have been used in a routine way in the study and analysis of new absorption spectra, some of which are displayed in Fig. 3. All the spectra shown were recorded on a $2 \mathrm{~m}$ grazing incidence spectrograph. The absorption spectrum of freon [14] in the 6-7 $\mathrm{nm}$ range and that of methyl iodide [15] in the 10-25 nm range were recorded by simply introducing the appropriate gas into the spectrograph. No differential pumping system was used, so the gases were at the same pressure in both the target chamber and spectrograph. Such a simple technique is only made possible by the insensitivity of the laser-produced continuum source to ambient pressure.

The absorption spectrum of $\mathrm{Li}^{+}$is of particular interest as it was obtained using the laser-produced continuum in a time-resolved experiment [16]. The laser beam was divided optically into two parts, one of which created the absorbing lithium plasma, while the other produced the background continuum-emitting plasma via an optical delay. The spatial resolution attainable due to the point-like nature of the continuum source is particularly evident in the absorption structure at $17 \mathrm{~nm}$. The asymmetric spectral features at $8 \mathrm{~nm}$ correspond to two-electron autoionizing transitions and illustrate the effectiveness of the two plasma techniques in studying ionic absorption.

In the normal incidence region continua generated on lutetium and ytterbium targets have been used with photoelectric detection to study the Lyman $\alpha$ profile in a $Z$-pinch discharge [17].

\section{B. Radiometric Standard}

The peak emission of the continuum is known to vary slower than linearly with incident laser power. Furthermore, for laser fluxes $>10^{10} \mathrm{~W} \cdot \mathrm{cm}^{-2}$ it is relatively insensitive to increases in flux of almost an order of magnitude provided the incident laser energy remains constant. Consequently, the precise focusing conditions and laser mode structure do not greatly affect the continuum output. As a result, laser-produced continua have been proposed [18] as UV radiometric standards, and, in particular, as transfer standards [9]. At present the only available calibration source for the soft X-ray region is the synchrotron which, although providing an absolute standard, has the disadvantage to most experimenters of being inaccessible and expensive, whereas the laser-produced continuum could provide a cheap in situ method of calibration source.

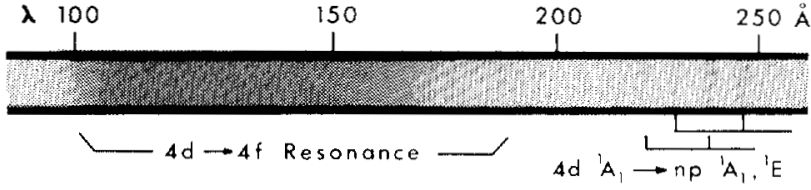

(a)

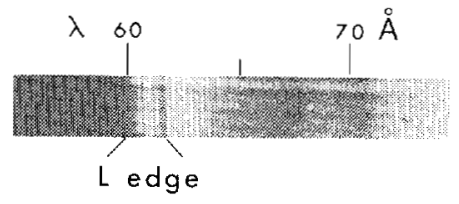

(b)

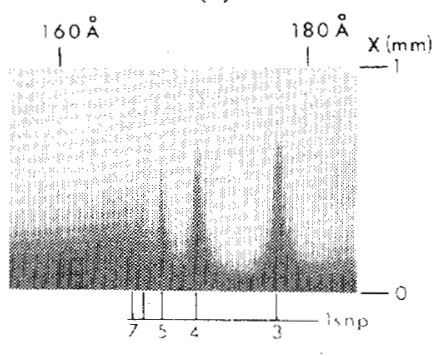

(c)

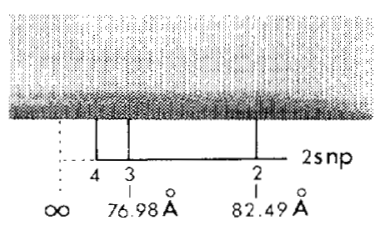

(d)

Fig. 3. Examples of absorption spectra obtained with laser-produced plasmas. (a) $L$ edge absorption of chlorine in freon; hafnium continuum [14]. (b) Giant $4 d-(\epsilon, 4) f$ resonance in methyl iodide; samarium continuum. (c) Ionic absorption: the principle series of $\mathrm{Li}^{+}$ observed in a dual plasma time-correlated experiment [16]. The vertical scale gives the distance from the surface of the lithium target. The experiment illustrates the use of the continuum source to provide spatial resolution. The background was generated on a tungsten target which, although dominated by lines at longer wavelengths, provides useful continuum in the region shown. (d) Autoionizing resonances $\left(1 s^{21} S-2 s n p^{1} P\right)$ in the absorption spectrum of $\mathrm{Li}^{+}[16]$.

\section{Calibration Testing of UV Soft X-Ray Detection Systems}

Even if an absolute calibration is not required, for example, in testing the long term stability of XUV detection systems, the reproducibility of the laser-produced plasma source makes it suitable for relative intensity measurements. The installation of such a system in inertial confinement devices has been suggested for the monitoring of soft $\mathrm{X}$-ray radiation losses-an essential prerequisite to their successful operation.

\section{Far UV/Soft X-Ray Fluorescence}

The intensity, wide spectral coverage, reproducibility, and short pulse duration of the laser-produced continuum source makes it suitable for the study of the fluorescence efficiency of materials as a function of irradiation wavelength. Two of the present authors (Kennedy and O'Sullivan) are currently using this technique to investigate the luminescence of newly developed glasses under far UV/soft X-ray (200-3 nm) excitation. Measurements of relative luminescent efficiency, decay time, and spectral emission as functions of base glass composition and activator type are being performed. 


\section{E. Study of Irradiation Damage}

The very high irradiance obtainable with laser-produced plasmas means that they can be used to study the deterioration in the performance of materials when subject to far UV/soft X-ray radiation. When placed $1 \mathrm{~cm}$ away from a laser-produced plasma the spatial irradiance at the sample can be as high as $2 \times 10^{4} \mathrm{~W} \cdot \mathrm{cm}^{-2} \cdot \mathrm{nm}^{-1}$ at $12 \mathrm{~nm}$ and the total irradiance between the wavelength limits of $3 \mathrm{~nm}$ and $200 \mathrm{~nm}$ may be greater than $2 \times 10^{6} \mathrm{~W} \cdot \mathrm{cm}^{-2}$.

\section{F. Transmission/Reflection Measurements}

Because of its stability and wide spectral coverage, the laserproduced plasma continuum source can be used in transmission or reflectance studies throughout the spectral range extending from 3 to $200 \mathrm{~nm}$.

\section{ACKNOWLEDGMENT}

Some of the work described here (the design of the automatic light source and the irradiance measurements) was carried out while two of the authors (P. K. Carroll and G. O'Sullivan) were visiting the Institute of Physical Science and Technology, University of Maryland, College Park, MD. We are grateful to Professor M. L. Ginter for helpful discussions and to R. Naber, G. Jellison, and J. Robinson for help in the light source construction.

\section{REFERENCES}

[1] P. K. Carroll, E. T. Kennedy, and G. O'Sullivan, "New continua for absorption spectroscopy from 40 to $2000 \AA$," Opt. Lett., vol. 2, pp. 72-74, 1978.

[2] - "Laser-produced continua for absorption spectroscopy in the vacuum ultraviolet and XUV," Appl. Opt., vol. 19, pp. 1454$1462,1980$.

[3] R. C. Fawcett, "Classifications in the period between calcium and iron of emission lines isoelectronic with those of carbon, nitrogen, oxygen and fluorine," J. Phys. B., vol. 4, pp. 981-985, 1971.

[4] G. A. Doschek, U. Feldman, R. D. Cowan, and L. Cohen, "Transitions $2 s^{2} 2 p^{k}-2 s 2 p^{k+1}$ of the FI, OI and NI isoelectronic sequences," vol. 188, pp. 417-422, 1974.

[5] G. O'Sullivan, P. K. Carroll, T. J. Mcllrath, and M. L. Ginter, "New continuum light source for VUV applications," Appl. Opt., vol. 20, pp. 3043-3046, 1981.

[6] G. O'Sullivan, J. R. Roberts, W. R. Ott, J. M. Bridges, T. L. Pittman, and M. L. Ginter, "Spectral irradiance calibration of continuum emitted from rare earth plasmas," Opt. Lett., vol. 7,pp. 31-33, 1982.

[7] C. Breton and R. Papoular, "Vacuum-UV radiation of laserproduced plasmas," Opt. Soc. Amer., vol. 63, pp. 1225-1232, 1972.

[8] P. Nicolosi, E. Jannitti, and G. Tondello, "Soft X-ray emission of continua from laser-produced plasmas," Appl. Phys, vol. B26, p. 117,1981

[9] M. Kühne, "Radiometric comparison of a laser-produced plasma and a BRV-source plasma at normal incidence," Appl. Opt., vol. 21, pp. 2124-2128, 1980 .

[10] D. Colombant and G. F. Tonon, "X-ray emission in laser produced plasmas," J. Appl. Phys., vol. 44, pp. 3524-3527, 1973.

[11] G. O'Sullivan and P. K. Carroll, " $4 d-4 f$ emission resonances in laser-produced plasmas," J. Opt. Soc. Amer., vol. 71, pp. 227230,1981

[12] P. K. Carroll and G. O'Sullivan, "Ground-state configurations of ionic species I through XVI for $Z=57-74$ and the interpretation of $4 d-4 f$ emission resonances in laser-produced plasmas," Phys. Rev. $A$, vol. 25, pp. 275-286, 1982.

[13] G. O'Sullivan, "The origin of line-free XUV continuum emission from laser-produced plasmas of the elements $62 \leqslant Z \leqslant 74$," unpublished work.
[14] - "Chlorine $L$-edge absorption in $\mathrm{CCl}_{4}$ and $\mathrm{CCl}_{2} \mathrm{~F}_{2}$," J. Phys. $B$, vol. 15, pp. 2385-2390, 1982.

[15] - "The absorption spectrum of $\mathrm{CH}_{3} \mathrm{I}$ in the extreme VUV," J. Phys. B, vol. 15, pp. L237-L330, 1982.

[16] P. K. Carroll and E. T. Kennedy, "Doubly excited autoionization resonances in the absorption spectrum of $\mathrm{Li}^{+}$formed in a laserproduced plasma," Phys. Rev. Lett., vol. 38, pp. 1068-1071, 1977.

[17] C. G. Mahajan, E.A.M. Baker, and D. D. Burgess, "Use of a laserproduced plasma as a source of focused vacuum-ultraviolet radiation," Opt. Lett., vol. 4, pp. 283-284, 1979.

[18] P. K. Carroll, E. T. Kennedy, and G. O'Sullivan, "Laser-produced continua for absorption spectroscopy in the vacuum ultraviolet and the XUV," in Proc. 6th Int. Conf. VUV Radiat. Phys., vol. 3 , no. 56,1980 .

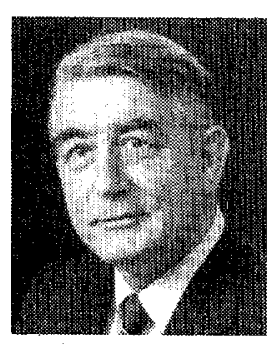

P. K. Carroll was born in Dublin, Ireland, on April 13, 1926. He received the B.Sc. degree from University College, Dublin in 1948, the Ph.D. degree in 1953, and the D.Sc. degree in 1975.

He lectured in the Departments of Physics at the Queen's University of Belfast (1951-1953) and University College, Dublin (1955-1960). He did postdoctoral work at the National Research Council of Canada, Ottawa (1953-1955) and later spent three years at the Laboratory of Molecular Structure and Spectra, University of Chicago, IL (1960-1964). In 1965 he returned to University College, Dublin where he is now Professor of Optical Physics. He has published about thirty papers on high-resolution molecular spectroscopy, which was his main interest for many years. More recently he has been working on the emission and absorption spectroscopy of hot dense plasmas, in particular laser-produced plasmas.

Dr. Carroll is a member of the Royal Irish Academy and the IUPAP Commission on Atomic and Molecular Physics and Spectra, and is a Fellow of the Institute of Physics (London).

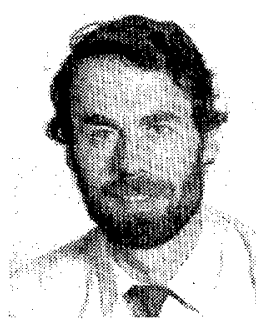

Eugene T. Kennedy was born in Boyle, Ireland, in 1948 . He received the B.Sc. degree in experimental physics and the Ph.D. degree from University College, Dublin in 1970 and 1977 , respectively.

From 1974 to 1977 he worked as a Research Associate in the Department of Physics, University College, Dublin, studying the emission and absorption spectroscopy of laser-produced plasmas in the UV/soft X-ray spectral regions. From 1977 to 1980 he held a lectureship at the Regional Technical College, Cork. From 1980 to the present he has been a Lecturer in the School of Physical Sciences, National Institute for Higher Education, Dublin where he has been Leader of the Laser/ Spectroscopy Research Group. His current interests are in the development of new glass scintillators for the vacuum ultraviolet and the use of laser-produced plasmas to study the absorption spectra of ions.

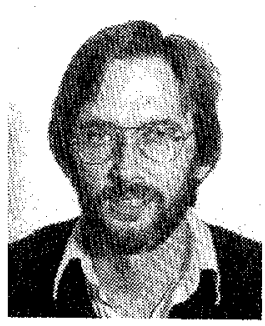

Gerard O'Sullivan was born in Dublin, Ireland, on September 25, 1953. He received the B.Sc. degree in experimental physics in 1975 and the Ph.D. degree in 1980 from University College, Dublin.

From 1980 to 1981 he worked as a Postdoctoral Research Fellow at the Department of Physics, University College, Dublin on the spectroscopy of laser-produced plasmas and applications of the laser-produced continuum to XUV absorption studies on polyatomic molecules. During this time he also spent brief periods at the U.S. National Bureau of Standards, Washington, DC and at the University of Maryland, College Park, MD, engaged in work on intensity calibration of laser-produced plasma emission in the VUV. From 1981 to the present he has been a Lecturer in the School of Physical Sciences, National Institute for Higher Education, Dublin. His current research is devoted to the analysis of spectra of highly ionized, high $Z$ atoms produced in laser plasmas. 